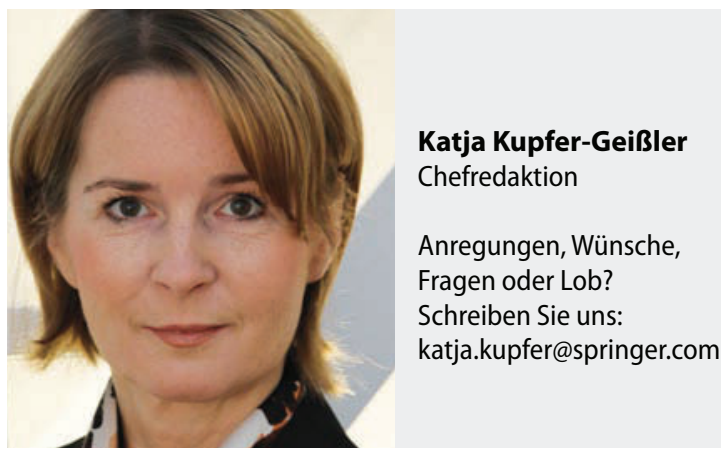

\title{
Der Jolie-Effekt
}

A ls wir diese HEILBERUFE-Ausgabe planten, Aahnten wir natürlich nicht, dass im Vorfeld ausgerechnet durch jemanden wie Angelina Jolie das Thema "Mammakarzinom" so populär werden würde. Doch nun wird die von ihr durchgeführte prophylaktische Brustentfernung mit einem Schlag weltweit besprochen. Die Mastektomie wird nicht mehr nur in Fachmedien diskutiert, sondern ist als Thema in Frauenzeitschriften genauso präsent wie in Talkshows. In den Kliniken spricht man mittlerweile vom Jolie-Effekt, denn die Anfragen nach umfassender Aufklärung häufen sich. Viele Frauen trauen sich jetzt, ihre Ärzte darauf anzusprechen viele Männer betroffener Frauen ahnen nun, dass eine solche Brustentfernung nicht mehr den Anschein einer Kriegsverstümmelung haben muss - denn sicher werden wir die Hollywood-Schauspielerin auch weiterhin dekolletiert über rote Teppiche schreiten sehen. Damit ist zwar das Bewusstsein aller dafür geschärft, dass es bei Brustkrebs einen familiären Hintergrund geben kann. Nicht vergessen werden aber darf, dass die prophylaktische Mastektomie nur ein gangbarer Weg und keine Pauschallösung ist. Und wenn sich die betroffenen Frauen für diesen Weg entscheiden, dann nur nach einer umfangreichen medizinischen Beratung und sicher zahlreichen weiteren Gesprächen auch mit den entsprechend geschulten Pflegekräften.

Neben dem PflegeKolleg „Mammakarzinom“ (Seite 15) bieten wir Ihnen mit „Krebstherapie: Folgen managen“ (Seite 29) und „Psyche \& Krebs“ (Seite 47) zwei weitere Möglichkeiten, Ihr Wissen zur onkologischen Pflege zu vertiefen und durch Ihre Teilnahme an der Fernfortbildung wertvolle Fortbildungspunkte zu sammeln.

Auch wenn wir nach viel Kälte und der großen Flut dieser Tage unter extremer Hitze stöhnen: Genießen Sie Ihren Sommer. Wir sehen uns im September wieder.

Ihre

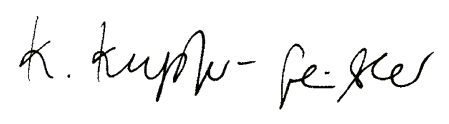

\section{HEILBERUFE SCIENCE SYMPOSIUM}

\section{Call for Abstracts}

Im Rahmen des 11. Gesundheitspflege-Kongresses (15.-16.11.2013) in Hamburg findet zum vierten Mal das HeilberufeSCIENCE-Symposium statt. In Vorträgen und einer Poster-Session werden aktuelle wissenschaftliche Beiträge aus Pflegemanagement, Pflegepädagogik, Pflegewissenschaft, Public Health, Gerontologie und Altenhilfe präsentiert.

Bis 31.8.2013 können die Abstracts zu wissenschaftlichen Studien, Abschlussarbeiten etc. - orientiert an den Kongressthemen oder zu einem frei gewählten Thema eingereicht werden. Schwerpunkte des Kongresses sind:
- Führungskräfte - Kompetenzen, Aufgaben, Förderung

- Die Zukunft der studierten Pflegekraft

$\checkmark$ Neue Konzepte in der Onkologie

- Älter werden im Pflegeberuf

- Demenzsensibel pflegen

- Organspende: Recht und Ethik

- Arbeit in multikulturellen Teams

Informationen zum Abstract und zum Kongress:

www.heilberufe-kongresse.de

\section{Call for Abstracts}

4. HeilberufeSCIENCESymposium

SpringerMedizin 